\title{
On Diameters of Uniformly Rotating Stars
}

\author{
Sagun Chanillo, YanYan Li \\ Department of Mathematics, Rutgers University, New Brunswick, NJ 08903, USA
}

Received: 24 August 1993/in revised form: 14 February 1994

\begin{abstract}
In this paper we study the compressible fluid model of uniformly rotating starts. It was proved in [Li] that for a given mass, there exists an equilibrium solution to the problem if the angular velocity is less than a certain constant. On the other hand for large angular velocities there is no equilibrium solution. In this paper we give an a-priori bound on diameters and the number of connected components of white dwarfs.
\end{abstract}

\section{Intrtoduction, Main Results, and Notation}

The object of our study in this paper are models of rotating white dwarf stars with a prescribed angular velocity about an axis. We will henceforth denote the angular velocity by $\omega$. The principal problem that we address in this paper is to determine a-priori bounds for the support of the relative equilibrium form of a homogeneous, gravitating and compressible mass of fluid when rotating about a fixed axis (which we will from now on select to be the z-axis) with constant angular velocity.

On the incompressible model of uniformly rotating stars (i.e. with constant angular velocity) there has been a tremendous amount of work. The first instance was Maclaurin (1742) who produced a family of exact solutions for the problem. In fact these spheroids as they are known obey the identity,

$$
\frac{\omega^{2}}{\pi G \rho}=2 \frac{\left(1-e^{2}\right)^{\frac{1}{2}}}{e^{3}}\left(3-2 e^{2}\right) \sin ^{-1} e-\frac{6}{e^{2}}\left(1-e^{2}\right),
$$

where $G$ is the gravitational constant, $\rho$ the density, taken to be a constant and $e$ the eccentricity. It is understood that these spheroids are ellipsoids with the z-axis as their symmetry axis (see e.g. [L]). It was observed by Thomas Simpson (1743) that (0.1) has a curious property. As $\omega \rightarrow 0$ we are led to two solutions, one a small perturbation of a ball $(e \rightarrow 0)$ as expected, and another a highly flattened ellipsoid $(e \rightarrow 1)$, where the flattening is in the $z$-direction. Since then there have been numerous investigations by Riemann, Jacobi, Darwin, Poincaré, H. Cartan and Chandrasekhar, where other families have been found, bifurcation sequences studied, and the stability and instability determined. Perturbation methods for approximating 
solutions have also been developed. The details are to be found in the treatises by Chandrasekhar [C2], Kopal[K], Poincaré[P], Wavre[W] and Lamb[L]. A historical overview is presented in Chanderasekhar's article [C3].

In the case of white dwarfs the stellar material by virture of the uncertainty principle and Pauli's exclusion principle exercises a ground state pressure which depends on the local density. This pressure in a non-rotating white dwarf is the sole local balance for the gravitational force as the star has no more nuclear fuel to burn to supply additional thermal and radiation pressure gradients. If the gravitational force is too large one has gravitational collapse leading to Chandrasekhar's celebrated mass-radius relation [C1] and [C4]. Well-known facts from quantum statistics reproduced for e.g. in Chap. 10 of Chandrasekhar's book [C1], shows that the pressure $f(\rho)$ obeys the asymptotics,

$$
\begin{gathered}
f(\rho) \sim C_{1} \rho^{5 / 3}-C_{2} \rho^{7 / 3}+O\left(\rho^{3}\right), \quad \rho \rightarrow 0, \\
f(\rho) \sim C_{3} \rho^{4 / 3}-C_{4} \rho^{2 / 3}+\cdots, \quad \rho \rightarrow \infty .
\end{gathered}
$$

Here $C_{i}>0(1 \leqq i \leqq 4)$, their precise values and higher asymptotics are noted as Eqs. (19), (21), (24) and (25) in Chap. 10 of [C1]. We re-iterate that the symbol $\rho$ will always stand for the density of the stellar material.

The compressible fluid model of rotating stars with prescribed constant angular velocity was investigated earlier by one of us, Y.Y. Li [Li] who showed that for a given mass, there exists an equilibrium solution to the problem if the angular velocity is less than a certain constant. These solutions correspond to local minimizers among axisymmetric configurations of the functional $J(\rho)$ introduced below in (0.4.). On the other hand for large angular velocities he showed there is no equilibrium solution. Auchmuty and R. Beals [AB1-2] have proved the existence of equilibrium solutions if the angular velocity satisfies certain decay assumptions.

There is another model which has been the focus of study. There one prescribes the angular momentum per unit mass, instead of the angular velocity. See Auchmuty and R. Beals [AB1], Friedman and Turkington [FT1-3]. Here our focus shall be the constant angular velocity case. The regularity and shape of the free boundary for this model has been investigated by Caffarelli and Friedman [CF1].

We now fix our notation. We will denote points in $\mathbb{R}^{3}$ by $\xi$ and $\eta$, with $\xi, \eta=(x, y, z)$. We say that $\rho$ is axisymmetric if $\rho(x, y, z)=\rho\left(x^{\prime}, y^{\prime}, z\right)$ for all $x^{2}+y^{2}=\left(x^{\prime}\right)^{2}+\left(y^{\prime}\right)^{2}$. In that case, we would simply use the notation $\rho(r, z)$ for $\rho(x, y, z)$, where $r=\sqrt{x^{2}+y^{2}}$. We assume that $\rho$ is continuous and moreover $\rho \in L^{1} \cap L^{\infty}$. We normalize the total mass of the fluid so that $\int_{\mathbb{R}^{3}} \rho=1$. We denote the gravitational potential by $B \rho$ where,

$$
B \rho(\xi)=\int_{\mathbb{R}^{3}} \frac{\rho(\eta)}{|\xi-\eta|} d \eta .
$$

Let $f:[0, \infty) \rightarrow[0, \infty)$ be an absolutely continuous, strictly increasing function, such that for some $\gamma$, in the range, $1<\gamma<\infty$,

$$
\lim _{t \rightarrow 0^{+}} f(t) t^{-\gamma}=C_{1}>0, \liminf _{t \rightarrow \infty} f(t) t^{-4 / 3}=M_{0}>3 / 2(4 \pi)^{1 / 3} .
$$

We do allow $M_{0}$ to be infinite. In view of the fact that the total mass has been normalized to be unity, $(0.3)$ corresponds to the total mass being strictly less than 
the Chandrasekhar mass corresponding to the second of the two relations in (0.2). In view of $(0.2)$ we assume that the equation of state for the pressure $p$ is given by $p=f(\rho)$. Note $(0.2)$ tells us that the physically important case is when $\gamma=\frac{5}{3}$. We also define a smoothed out version of $f$, whose properties we develop in Sect. 1. Precisely we define,

$$
A(s)=s \int_{0}^{s} f(t) t^{-2} d t
$$

Set

$$
J(\rho)=\int_{\mathbb{R}^{3}} A(\rho)-\frac{1}{2} \int_{\mathbb{R}^{3}} \omega^{2} r^{2} \rho-\frac{1}{2} \int_{\mathbb{R}^{3}} \rho B \rho .
$$

In the non-rotating case $(\omega=0)$ Lieb and Yau have obtained the well known semiclassical theory as a limit of quantum mechanics. See [LY].

Definition. Let $\rho \in C_{c}^{0}\left(\mathbb{R}^{3}\right)$ be a nonnegative function with $\int_{\mathbb{R}^{3}} \rho=1$. We say that $\rho$ is a critical point of the functional $J(\rho)$ if for some positive constants $\varepsilon, \delta>0$ and any family $\left\{\rho_{t}\right\}(-\delta<t<\delta)$ satisfying

(1) $\rho_{t} \in C_{c}^{0}\left(\mathbb{R}^{3}\right)$ is nonnegative and the map $t \rightarrow \rho_{t}$ is continuous from $(-\delta, \delta)$ to $C^{0}\left(\mathbb{R}^{3}\right)$. Furthermore supp $\rho_{t}(-\delta<t<\delta)$ lies in the $\varepsilon$ neighborhood of supp $\rho, \rho_{0}=\rho$ and $\int_{\mathbb{R}^{3}} \rho_{t}=1$.

(2) $J\left(\rho_{t}\right)$ belongs to the class $C^{1}(-\delta, \delta)$.

(3) The famiy $\left\{\rho_{t}\right\}$ is uniformly bounded in $L^{\infty}$.

We have,

$$
\left.\frac{d}{d t} J\left(\rho_{t}\right)\right|_{t=0}=0 .
$$

If $\rho$ is also axisymmetric and all the above are restricted to the axisymmetric class, we say that $\rho$ is a critical point among axisymmetric configurations of the functional $J(\rho)$.

Since the total mass has been constrained to be unity we see that the critical points of the functional $J(\rho)$, for some negative constant $\lambda(\omega)$, satisfy the pair of equations,

$$
\begin{aligned}
& \int_{\mathbb{R}^{3}} \rho=1, \quad \rho \geqq 0 \text { is continuous, } \\
& \quad A^{\prime}(\rho)-\frac{1}{2} \omega^{2} r^{2}-B \rho=\lambda(\omega), \text { on } \rho>0 .
\end{aligned}
$$

(0.6) will be referred to as the equilibrium equations. We will also suppress the dependence of the Lagrange parameter $\lambda$ on $\omega$. We can now state our theorems.

Theorem 1. Let $\omega \geqq \omega_{0}>0$. Let $\rho$ be a critical point of $J(\rho)$ or a critical point among axisymmetric configurations of $J(\rho)$. Then there is a ball $B_{\sigma}$, centered at some point on the $z$-axis and having a radius $\sigma=\sigma\left(\omega_{0}\right)$ such that the support of $\rho$ is contained in $B_{\sigma}$.

It is reasonable to believe that we do need a lower bound on the angular velocity $\omega$ in order to obtain the a-priori bound of the support of $\rho$ since in the incompressible case the Maclaurin spheroids (0.1) flatten out as $\omega \rightarrow 0$. 
For the local minimizers of the functional $J(\rho)$, Li [Li] had obtained information about the free boundary of the star following Caffarelli and Friedman's [CF1] work. For the critical points of $J(\rho)$, the description of the free boundary seems to be a difficult and interesting problem. In the following we provide an upper bound of the number of connected components of the set where $\rho>0$ uder the hypothesis that $\omega \geqq \omega_{0}>0$.

Theorem 2. Let $\omega \geqq \omega_{0}>0$ and $\rho$ be a critical point of $J(\rho)$ or a critical point among axisymmetric configurations of $J(\rho)$. Then there is a number $k=k\left(\omega_{0}\right)$ such that the number of connected components of the set where $\rho>0$, is at most $k$.

Remark 2. We will only establish Theorem 1 and Theorem 2 for $\rho$ a critical point of $J(\rho)$. For $\rho$ a critical point among axisymmetric configurations of $J(\rho)$, one only needs to make some slight modifications.

Both Theorems 1 and 2 are proved by arguments based on contradiction. Thus we have no explicit dependence of $\sigma$ or $k$ on $\omega$ or $\omega_{0}$. It is interesting to obtain such a dependence. Furthermore, in this paper we do not address the important question of stability. Stability questions are discussed, for example, in [C2].

In what follows $C_{1} \geqq 1$ will denote a generic constant independent of $\omega$ and $\omega_{0}$, and $C_{0} \geqq 1$ a constant that does depend on $\omega_{0}, C_{0}=C_{0}\left(\omega_{0}\right)$. The plan of the paper is as follows. In Sect. 1 we collect some basic inequalities for the gravitational potential. In Sect. 2 we establish a-priori bounds on the support of $\rho$ in the $r$ direction. In Sect. 3 we further establish a-priori bounds on the support of $\rho$ in the $\mathrm{z}$-axis direction. This completes the proof of Theorem 1. The rest of Sect. 3 is devoted to proving Theorem 2 .

\section{Some Calculus Inequalities}

In this section we collect some inequalities which we will use in Sects. 2 and 3.

Lemma (1.1).

$$
\|B \rho\|_{\infty} \leqq 3 / 2(4 \pi)^{1 / 3}\|\rho\|_{1}^{2 / 3}\|\rho\|_{\infty}^{1 / 3} .
$$

Proof. Split the integral for $B \rho$ as follows,

$$
\begin{aligned}
|B \rho(\xi)| & \leqq \int_{|\zeta-\eta| \leqq \delta} \frac{\rho(\eta)}{|\xi-\eta|} d \eta+\int_{|\xi-\eta|>\delta} \frac{\rho(\eta)}{|\xi-\eta|} d \eta \\
& \leqq 2 \pi \delta^{2}\|\rho\|_{\infty}+\delta^{-1}\|\rho\|_{1} .
\end{aligned}
$$

If we select $\delta=\left(\frac{\|\rho\|_{1}}{4 \pi\|\rho\|_{\infty}}\right)^{1 / 3}$ we get the conclusion of the lemma.

We remark that the estimates following (2.7) can be need to show that the factor of $3 \rho$ above can be replaced by the sharp value $3^{2 / 3} / 2$.

\section{Lemma (1.2).}

$$
\|\nabla B \rho\|_{\infty} \leqq C_{1}\|\rho\|_{1}^{1 / 3}\|\rho\|_{\infty}^{2 / 3}
$$


Proof. The proof is identical to that of Lemma (1.1). Note,

$$
\begin{aligned}
|\nabla B \rho(\xi)| & \leqq C_{1} \int_{|\zeta-\eta|<\delta} \frac{\rho(\eta)}{|\xi-\eta|^{2}} d \eta+C_{1} \int_{|\zeta \xi-\eta| \geqq \delta} \frac{\rho(\eta)}{|\xi-\eta|^{2}} d \eta \\
& \leqq C_{1}\|\rho\|_{\infty} \delta+C_{1}\|\rho\|_{1} \delta^{-2} .
\end{aligned}
$$

Select $\delta=\left(\frac{\|\rho\|_{1}}{\|\rho\|_{\infty}}\right)^{1 / 3}$ to get the conclusion of the lemma.

We now collect some helpful facts about the function $A(s)$ which was defined in the introduction.

Lemma (1.3). Let $A(s)$ be as in Sect. 0. Then,

(a) $A(s)$ is strictly increasing, and $f\left(\frac{s}{2}\right) \leqq A(s) \leqq C_{1} f(s)$.

(b) $A^{\prime}(s)$ is continuous, strictly increasing and $\lim _{\inf _{s \rightarrow \infty}} A^{\prime}(s) s^{-1 / 3}>3 / 2(4 / \pi)^{1 / 3}$.

(c) Furthermore, $A^{\prime}(s) s^{1-\gamma} \geqq 1 / C_{1}>0$ for $s \leqq s_{1}$.

(d) The inverse function $\Phi(t)$ to $A^{\prime}(s)$ exists and $\Phi(t) \leqq C_{1} t^{1 /(\gamma-1)}$ as $t \rightarrow 0^{+}$.

Proof. By changing variables, we can express $A(s)$ as

$$
A(s)=\int_{0}^{1} f(s t) t^{-2} d t .
$$

Since $f$ is strictly increasing it follows that $A$ is strictly increasing. Further by the definition of $A$,

$$
A(s) \geqq s \int_{\frac{s}{2}}^{s} f(t) t^{-2} d t \geqq f\left(\frac{s}{2}\right) .
$$

The remaining inequality of part (a) is also an easy exercise.

Note now,

$$
A^{\prime}(s)=\frac{f(s)}{s}+\int_{0}^{s} f(t) t^{-2} d t .
$$

This yields $A^{\prime}(s) \geqq f(s) s^{-1}$ and thus for $\liminf _{s \rightarrow \infty} A^{\prime}(s) s^{-1 / 3}>$ $3 / 2(4 \pi)^{1 / 3}$ and likewise for $s \leqq s_{1}$ we get $A^{\prime}(s) s^{1-\gamma} \geqq 1 / C_{1}>0$. To see that $A^{\prime}(s)$ is strictly increasing, we may integrate by parts, to get

$$
A^{\prime}(s)=\int_{0}^{s} f^{\prime}(t) t^{-1} d t
$$

Since $f$ is strictly increasing it follows immediately from the identity above that $A^{\prime}(s)$ is strictly increasing. (b) and (c) are established.

Since $A^{\prime}(s)$ is continuous and increases strictly, $\Phi(t)$ the inverse function exists. Now $s=\Phi\left(A^{\prime}(s)\right) \geqq \Phi\left(C_{1}^{-1} s^{\gamma-1}\right)$. Setting $t=C_{1}^{-1} s^{\gamma-1}$ we get (d).

\section{The Support Estimates in the $r$-Direction}

Let $d=\sup \left\{r \mid r=\left(x^{2}+y^{2}\right)^{1 / 2}\right.$, for some $\left.(x, y, z) \in \operatorname{supp} \rho\right\}$. 
Lemma (2.1). Let $\rho(x, y, z)$ be any solution of $(0.6)$. Let $\omega \geqq \omega_{0}>0$. Then there exists a number $d_{0}=d_{0}\left(\omega_{0}\right)<\infty$ such that $d \leqq d_{0}$.

Proof. We prove this lemma by contradiction. Assume no such $d_{0}$ exists. First note that for $\rho(x, y, z)$ satisfying $(0.6)$, we certainly have $d<\infty$. For if not one can find a sequence of points $\left(x_{n}, y_{n}, z_{n}\right)$, such that $\rho\left(x_{n}, y_{n}, z_{n}\right)>0$ and $r_{n}=\sqrt{\left(x_{n}\right)^{2}+\left(y_{n}\right)^{2}} \rightarrow \infty$. Since $\int_{\mathbb{R}^{3}} \rho=1$, we can assume that in fact $\rho\left(x_{n}, y_{n}, z_{n}\right) \rightarrow 0$ as $r_{n} \rightarrow \infty$. Thus $A^{\prime}(\rho)\left(x_{n}, y_{n}, z_{n}\right) \rightarrow 0$. However $\rho$ satisfies (0.6) and so we see that as $r_{n} \rightarrow \infty$, it forces $\lambda=-\infty$. This is a contradiction.

Now, for $\omega$ fixed, select a sequence $r_{n} \rightarrow d$, such that $\rho\left(x_{n}, y_{n}, z_{n}\right)>0$. From $(0.6)$ we see,

$$
A^{\prime}(\rho)\left(x_{n}, y_{n}, z_{n}\right)-\frac{1}{2} \omega^{2} r_{n}^{2}-B \rho\left(x_{n}, y_{n}, z_{n}\right)=\lambda
$$

But $A^{\prime}(\rho)\left(x_{n}, y_{n}, z_{n}\right) \rightarrow 0$ as $r_{n} \rightarrow d$. Hence

$$
\lambda+\frac{1}{2} \omega^{2} d^{2} \leqq 0
$$

Using (0.6), (2.2) and the fact that $A^{\prime}(\rho) \geqq 0$, we have, at all points where $\rho(x, y, z)>0$, that

$$
\begin{aligned}
-B \rho \leqq A^{\prime}(\rho)-B(\rho) & =\lambda+\frac{1}{2} \omega^{2} r^{2} \\
& \leqq \frac{1}{2} \omega^{2} r^{2}-\frac{1}{2} \omega^{2} d^{2} .
\end{aligned}
$$

We have now arrived at the estimate,

$$
\frac{1}{2} \omega^{2}(d+r)(d-r) \leqq B \rho
$$

from which it follows for $\omega \geqq \omega_{0}$, that

$$
d-r \leqq \frac{C_{1}\|B \rho\|_{\infty}}{\omega_{0}^{2} d}
$$

Further from (0.6) and (2.2) we also have on the set $\rho>0$,

$$
A^{\prime}(\rho)=\lambda+\frac{1}{2} \omega^{2} r^{2}+B \rho \leqq B \rho
$$

Thus it follows that,

$$
\left\|A^{\prime}(\rho)\right\|_{\infty} \leqq\|B \rho\|_{\infty} .
$$

But Lemma (1.1) yields $\|B \rho\|_{\infty} \leqq 3 / 2(4 \pi)^{1 / 3}\|\rho\|_{\infty}^{1 / 3}$. This fact and (2.4) together yields $\left\|A^{\prime}(\rho)\right\|_{\infty} \leqq 3 / 2(4 \pi)^{1 / 3}\|\rho\|_{\infty}^{1 / 3}$. It follows from part (b) of Lemma (1.3) that

$$
\|\rho\|_{\infty} \leqq C_{1} .
$$


Combining (2.5) with Lemma (1.1) yields $\|B \rho\|_{\infty} \leqq C_{1}$. Inserting these estimates into (2.3), we get for $\omega \geqq \omega_{0}$,

$$
\omega_{0}^{2}(d-r) \leqq \frac{C_{1}}{d}
$$

We will set,

$$
\varepsilon=\sup \left\{d-r \mid \text { for some } \rho(x, y, z)>0, r=\sqrt{x^{2}+y^{2}}\right\} .
$$

From the inequality above notice that if $d \geqq d_{1}\left(\omega_{0}, C_{1}\right)$, one forces $\varepsilon \leqq \frac{1}{2}$. We shall thus assume this with no loss of generality. We write our conclusion (2.3) and the one above in our new notation as,

and

$$
\varepsilon \leqq \frac{C_{1}\|B \rho\|_{\infty}}{\omega_{0}^{2} d}
$$

$$
\varepsilon \leqq \frac{C_{1}}{\omega_{0}^{2} d} .
$$

To proceed further we need to estimate $\|B \rho\|_{\infty}$. These estimates in conjunction with (2.3) will lead us to a contradiction if $d$ increases without bound. Notice at this point we have shown that the support of $\rho$ is contained in a cylindrical shell whose outer radius is $d$, the inner radius is $d-\varepsilon$ and has perhaps infinite length along the z-axis. Let

$$
S=\left\{(x, y, z) \mid d-\varepsilon<\sqrt{x^{2}+y^{2}}<d\right\} .
$$

It follows from the strong maximum principle that there exists $\xi$ belonging to the support of $\rho$, such that $\|B \rho\|_{\infty}=B \rho(\xi)$. Without loss of generality we can assume $\xi=(0, a, 0), d-\varepsilon<a<d$. Let $D_{l}=\left\{(x, y, z) \mid x^{2}+(y-a)^{2}+z^{2}<l^{2}\right\}$, where $l$ satisfies

$$
\|\rho\|_{\infty}\left|D_{l} \cap S\right|=1 \text {. }
$$

Set $E=D_{l} \cap S$ and $\tilde{\rho}=\|\rho\|_{\infty} \chi_{E}$. We have

$$
\begin{aligned}
B \rho(\xi)= & \int_{\mathbb{R}^{3}} \frac{\rho d x d y d z}{\left(x^{2}+(y-a)^{2}+z^{2}\right)^{1 / 2}} \\
= & \int_{E} \frac{\rho d x d y d z}{\left(x^{2}+(y-a)^{2}+z^{2}\right)^{1 / 2}}+\int_{E^{c}} \frac{\rho d x d y d z}{\left(x^{2}+(y-a)^{2}+z^{2}\right)^{1 / 2}} \\
= & \|\rho\|_{\infty} \int_{E} \frac{d x d y d z}{\left(x^{2}+(y-a)^{2}+z^{2}\right)^{1 / 2}}+\int_{E} \frac{\left(\rho-\|\rho\|_{\infty}\right) d x d y d z}{\left(x^{2}+(y-a)^{2}+z^{2}\right)^{1 / 2}} \\
& +\int_{E^{c}} \frac{\rho d x d y d z}{\left(x^{2}+(y-a)^{2}+z^{2}\right)^{1 / 2}} \\
= & B \tilde{\rho}(\xi)+\int_{E} \frac{\left(\rho-\|\rho\|_{\infty}\right) d x d y d z}{\left(x^{2}+(y-a)^{2}+z^{2}\right)^{1 / 2}}+\int_{E^{c}} \frac{\rho d x d y d z}{\left(x^{2}+(y-a)^{2}+z^{2}\right)^{1 / 2}} \\
\leqq & B \tilde{\rho}(\xi)+1 / l \int_{E}\left(\rho-\|\rho\|_{\infty}\right) d x d y d z+1 / l \int_{E^{c}} \rho d x d y d z \\
= & B \tilde{\rho}(\xi)+1 / l \int_{\mathbb{R}^{3}} \rho d x d y d z-1 / l\|\rho\|_{\infty}|E| \\
= & B \tilde{\rho}(\xi) .
\end{aligned}
$$


It follows that

$$
\|B \rho\|_{\infty} \leqq B \tilde{\rho}(\xi) .
$$

Clearly $l \geqq 1$ because of (2.5) and (2.7).

There are three cases.

Case $1 . l \leqq 10 d$.

Case 2. $10 d<l \leqq 100 d$.

Case 3. $l>100 d$.

In Case 1 , it is easy to see that

$$
C_{1}^{-1} l^{2} \varepsilon \leqq|E| \leqq C_{1} l^{2} \varepsilon .
$$

It follows from the above and (2.7) that

$$
1 / C_{1} \leqq l^{2} \varepsilon\|\rho\|_{\infty} \leqq C_{1} .
$$

It follows that

$$
\begin{aligned}
\|B \rho\|_{\infty} & \leqq B \tilde{\rho}(\xi) \\
& \leqq\|\rho\|_{\infty} \int_{E} \frac{d x d y d z}{\left(x^{2}+(y-a)^{2}+z^{2}\right)^{1 / 2}} \\
& \leqq C_{1}\|\rho\|_{\infty} \varepsilon l .
\end{aligned}
$$

The last inequality can be verified easily.

Thus, in view of (2.9),

$$
\|B \rho\|_{\infty} \leqq \frac{C_{1}}{l} .
$$

We also observe that combining (2.4) and Lemma (1.3) we have

$$
\|\rho\|_{\infty} \leqq C_{1}\|B \rho\|_{\infty}^{1 /(\gamma-1)} \text {. }
$$

Using (2.9) and (2.5) we have $\varepsilon^{-1} \leqq C_{1} l^{2}$. Using (2.10) and (2.6) we have $\varepsilon^{-1} \geqq C_{1}^{-1} \omega_{0}^{2} d l$. Hence

$$
l \geqq C_{1}^{-1} \omega_{0}^{2} d .
$$

On the other hand, substituting (2.6) in the left half of (2.9) yields

$$
l^{2} \geqq \frac{\omega_{0}^{2} d}{C_{1}\|\rho\|_{\infty}\|B \rho\|_{\infty}} .
$$

while from (2.11) and (2.10) we have $\|\rho\|_{\infty}\|B \rho\|_{\infty} \leqq C_{1} l^{-\gamma /(\gamma-1)}$. Hence

$$
d l^{(2-\gamma) /(\gamma-1)} \leqq C_{1} \omega_{0}^{2} \text {. }
$$

When $\gamma<2$, it follows from (2.12) and (2.13) that

$$
d \leqq C_{1} \omega_{0}^{2}
$$

When $\gamma \geqq 2,(2.12)$ shows that $d$ grows sublinearly with $l$; therefore in view of that fact we are considering Case $1: l \leqq 10 d$, a bound for $l$ and $d$ again follow from (2.13). 
In Case 2, it is easy to see that

$$
C_{1}^{-1} d^{2} \varepsilon \leqq|E| \leqq C_{1} d^{2} \varepsilon .
$$

It follows from the above and (2.7) that

$$
1 / C_{1} \leqq d^{2} \varepsilon\|\rho\|_{\infty} \leqq C_{1} .
$$

As in the derivation of (2.10), we can obtain

$$
\|B \rho\|_{x} \leqq \frac{C_{1}}{d} .
$$

Combining (2.14) with (2.6) we get $\varepsilon \leqq C_{0} d^{-2}$. It follows that in Case 2 we have $\|\rho\|_{\infty} \geqq 1 / C_{0}$. But from (2.11) it follows $\|B \rho\|_{\infty} \geqq 1 / C_{0}$. This contradicts (2.14) for large $d$.

In Case 3, it is easy to see that

$$
C_{1}^{-1} \varepsilon d l \leqq|E| \leqq C_{1} \varepsilon d l .
$$

It follows from the above and (2.7) that

$$
1 / C_{1} \leqq \varepsilon d l\|\rho\|_{\infty} \leqq C_{1} .
$$

It follows from (2.8) that

$$
\begin{aligned}
& \|B \rho\|_{\infty} \leqq\|\rho\|_{\infty} \int_{E} \frac{d x d y d z}{\left(x^{2}+(y-a)^{2}+z^{2}\right)^{1 / 2}}
\end{aligned}
$$

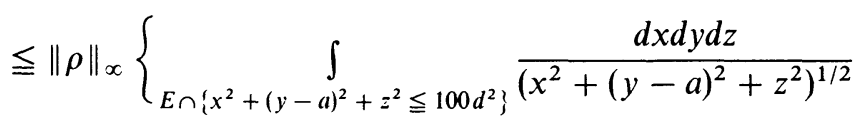

$$
\begin{aligned}
& \left.+\int_{E \cap\left\{x^{2}+(y-a)^{2}+z^{2} \geqq 100 d^{2}\right\}} \frac{d x d y d z}{\left(x^{2}+(y-a)^{2}+z^{2}\right)^{1 / 2}}\right\}
\end{aligned}
$$

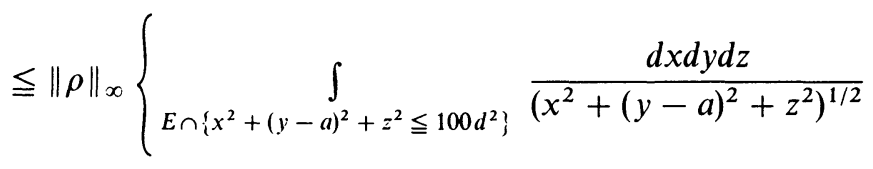

$$
\begin{aligned}
& \left.+\int_{\substack{(d-\varepsilon)^{2} \leqq x^{2}+y^{2} \leqq d^{2} \\
5 d \leqq z \leqq 2 l}} \frac{d x d y d z}{z}\right\} \\
& \leqq C_{1}\|\rho\|_{\infty} \varepsilon d \log \left(\frac{l}{d}\right) \text {. }
\end{aligned}
$$

The last inequality follows from estimates in Case 1 and some elementary calculation.

Thus

$$
\begin{aligned}
\|B \rho\|_{\infty} & \leqq C_{1}\|\rho\|_{\infty} \varepsilon d \log \left(\frac{l}{d}\right) \\
& \leqq C_{1}\|\rho\|_{\infty} \varepsilon d \log l
\end{aligned}
$$


It follows that in Case 3 we have

$$
\|B \rho\|_{\infty} \leqq \frac{C_{1} \log l}{l}
$$

From (2.15) and (2.11),

$$
\|\rho\|_{\infty} \leqq C_{1}\left(\frac{\log l}{l}\right)^{1 /(\gamma-1)} .
$$

From (2.6) and (2.15) we get,

$$
\varepsilon \leqq C_{0} \frac{\log l}{d l}
$$

Notice that it follows from (2.16) that in Case 3 we have

$$
\varepsilon \geqq \frac{1}{C_{1} d l}\left(\frac{l}{\log l}\right)^{1 /(\gamma-1)} .
$$

Combining the last two inequalities we get,

$$
\frac{C_{0}}{\log l} \leqq C_{1}\left(\frac{\log l}{l}\right)^{1 /(\gamma-1)} .
$$

This is clearly a contradiction if $d$ and hence $l$ increases without bound. Lemma (2.1) is fully established.

The next lemma is established in [Li] (see Theorem 3.2 there) under slightly different hypotheses. But the proof, after modification, clearly applies to our situation.

Lemma (2.17). Let $\rho$ satisfy (0.6) with $\omega \geqq \omega_{0} \geqq 0$. Then there exists a number $d_{1}=d_{1}\left(\omega_{0}\right)>0$ such that $d \geqq d_{1}$.

Sketch of the proof. We consider two cases. First we consider the case when $\varepsilon \geqq d / 2$. By Eq. (31) of [Li], we see for $\beta>2$,

$$
\|B \rho\|_{\infty} \leqq C_{1} d^{\beta} \text {. }
$$

Thus from (2.3) above, and the last inequality we get $\omega_{0}^{2} \leqq C_{1} d^{\beta-2}$. This immediately yields the conclusion of the lemma in this case. In the remaining case $\varepsilon \leqq \frac{d}{2}$, we use Eq. (36) of [Li] to see that,

$$
\omega_{0}^{2} \leqq C_{1}(\varepsilon d)^{\alpha}, \text { with } \alpha>0 .
$$

Using the fact that $\varepsilon \leqq \frac{d}{2}$, we see that $d \geqq C_{1} \omega_{0}^{1 / \alpha}$. The lemma follows.

We now deduce the following corollary.

Corollary (2.18). Let $\rho$ satisfy $(0.6)$. Then $\lambda \leqq-\frac{1}{2} \omega_{0}^{2} d_{1}^{2}$.

Proof. We see from (2.2) and Lemma (2.17) that

$$
\lambda+\frac{1}{2} \omega_{0}^{2} d_{1}^{2} \leqq 0
$$

The result follows. 


\section{The Suport Estimates in the z-Direction}

We shall prove the remaining part of Theorem 1 .

Lemma (3.1). Let $\rho$ be any critical point of $J(\rho)$. Let $\omega \geqq \omega_{0}>0$. Then there exists a number $M=M\left(\omega_{0}\right)$ and a point $\left(0,0, z_{1}\right)$ on the $z$-axis such that $\left|z-z_{1}\right| \leqq M$, for all $(x, y, z)$ in the support of $\rho$.

Proof. We first note that for (0.6) to hold, there exists some $\omega_{1}>0$ such that $\omega \leqq \omega_{1}$. This has been already established in [Li] under slightly different hypotheses on the function $f(\rho)$. The proof in [Li] can easily be modified to obtain the same upper bound on $\omega$ under our hypotheses. In fact the choice of $\delta$ given by Eq. (27) in [Li] is not necessary, and in fact, we can choose $\delta$ to be any small number such that $0<\delta<1$. The rest of the argument for Theorem (3.1) in [Li] carries over under the hypotheses in the current paper.

We begin the proof of Lemma (3.1) by defining horizontal slabs as follows. For $n \in \mathbb{Z}$ and $R^{\star} \geqq 2$ we define,

$$
Z_{n}^{\prime}=\left\{(x, y, z)|| z-2 n R_{\star} \mid \leqq R_{\star}\right\}
$$

and,

$$
Z_{n}=\left\{(x, y, z)|| z-2 n R_{\star} \mid \leqq 1\right\} .
$$

Fix now a number $\delta=\delta\left(\omega_{0}\right)>0$. Now notice that there exist at most $\delta^{-1}$ choices of $n$ for which $\int_{Z_{n}} \rho \geqq \delta$. This follows because $\int_{\mathbb{R}^{3}} \rho=1$. Denote the set of these values of $n$ by $D$. We now set $\mu=\frac{1}{2} \omega_{0}^{2} d_{1}^{2}$, (see Lemma (2.17) for the definition of $\left.d_{1}\right)$ and $\tau=\mu^{1 / 2} / \omega_{1}$. We claim that if $n \notin D$, then for appropriate choices of $\delta=\delta\left(\omega_{0}\right)$ and $R_{\star}\left(\omega_{0}\right)$ we have,

$$
B \rho(\xi) \leqq \frac{\mu}{2} \text { and }|\nabla B \rho(\xi)| \leqq \frac{1}{2} \omega_{0}^{2} \tau \text {, for all } \xi \in Z_{n} .
$$

We now verify (3.2). Using (2.5) and the Schwarz's inequality we get,

$$
\begin{aligned}
B \rho(\xi) & \leqq \int_{\eta \in Z_{n}^{\prime}} \frac{\rho(\eta)}{|\xi-\eta|}+\int_{\eta \notin Z_{n}^{\prime}} \frac{\rho(\eta)}{|\xi-\eta|} \\
& \leqq C_{1} \delta^{1 / 2}\left(\max \left\{R_{\star}, d_{0}\right\}\right)^{1 / 2}+\frac{C_{1}}{R_{\star}} .
\end{aligned}
$$

We now select $\delta$ and $\mathrm{R}_{\star}$ so that the expression on the right above is at most $\frac{1}{2} \mu$. The claim for $|\nabla B \rho|$ is proved in a similar fashion.

We next claim that $\rho \equiv 0$ in $Z_{n}$ for any $n \notin D$. We begin by showing that for $(x, y, z)$ in the support of $\rho$, and $(x, y, z) \in Z_{n}$ we have $r=\sqrt{x^{2}+y^{2}} \geqq \tau$. To see this we apply (0.6) and the fact that $A^{\prime}(\rho) \geqq 0$, to Cor. (2.18) to get,

$$
-\frac{1}{2} \omega^{2} r^{2}-B \rho \leqq \lambda \leqq-\mu
$$

But by (3.2) $B \rho \leqq \frac{\mu}{2}$, so,

$$
-\frac{1}{2} \omega^{2} r^{2} \leqq-\frac{\mu}{2}
$$

This implies at once that $r \geqq \tau$. 
Now set $u=\lambda+\frac{1}{2} \omega^{2} r^{2}+B \rho$. Suppose for some $\left(x_{0}, y_{0}, z_{0}\right) \in Z_{n}, n \notin D$ but $\rho\left(x_{0}, y_{0}, z_{0}\right) \neq 0$. We define $\Xi_{z_{0}}=\left\{r \mid \rho\left(x, y, z_{0}\right)>0,(x, y)=\frac{r}{r_{0}}\left(x_{0}, y_{0}\right)\right\}$. Since $\rho$ is continuous, the set $\Xi_{z_{0}}$ is open. Let $I_{z_{0}}$ be any maximal connected subset of $\Xi_{z_{0}}$. This $I_{z_{0}}$ is an interval and $\rho$ vanishes at its end-points. Because $A^{\prime}(\rho)=u, u$ also vanishes at the end-points of $I_{z_{0}}$. Thus by Rolle's theorem there is a point in the interior of $I_{z_{0}}$ where $\frac{\partial u}{\partial r}=0$. But by (3.2),

$$
\frac{\partial u}{\partial r}=\omega^{2} r+\frac{\partial}{\partial r}(B \rho) \geqq \omega_{0}^{2} \tau-|\nabla B \rho| \geqq \omega_{0}^{2} \tau-\frac{1}{2} \omega_{0}^{2} \tau>0 .
$$

Thus we see that $\rho \equiv 0$ in $Z_{n}$ for all $n \notin D$.

We now apply a deformation argument to prove the claims that follow.

(A) The slabs $Z_{n}^{\prime}$, for $n \in D$ are contiguous.

(B) If $n \notin D$ then $\rho \equiv 0$ for either $z \leqq 2 n R_{\star}$ or $z \geqq 2 n R_{\star}$.

Notice this immediately proves Lemma (3.1) since the claims show that $\rho$ is suported in a slab whose total width along the $\mathrm{z}$-axis is $4 R_{\star} \delta^{-1}$. In fact claim (B) implies claim (A), thus we will simply show (B). We apply our deformation argument to a family $\left\{\rho_{t}\right\}$ given by,

$$
\rho_{t}(x, y, z)=\rho_{t}^{1}(x, y, z)+\rho_{t}^{2}(x, y, z),
$$

where,

$$
\rho_{t}^{1}(x, y, z)= \begin{cases}\rho(x, y, z) & \text { if } z \leqq 2 n R_{\star} \\ 0, & \text { elsewhere }\end{cases}
$$

and,

$$
\rho_{t}^{2}(x, y, z)= \begin{cases}\rho(x, y, z+t), & \text { if } z>2 n R_{\star}, \\ 0, & \text { elsewhere } .\end{cases}
$$

It is easy to verify that because $\rho \equiv 0$ in $Z_{n}$ the family $\left\{\rho_{t}\right\}$ is an admissible family of variations for $\rho$. Recalling the fact that $\rho_{t}=\rho_{t}^{1}+\rho_{t}^{2}$ and $\rho \equiv 0$ in $Z_{n}$, we have, for $|t| \leqq \frac{1}{10}$,

$$
J\left(\rho_{t}\right)=J(\rho)-\int_{\mathbb{R}^{3}} \rho_{t}^{1} B\left(\rho_{t}^{2}-\rho_{0}^{2}\right) .
$$

By a change of variable it follows easily that,

$$
\begin{aligned}
J\left(\rho_{t}\right)= & J(\rho)-\int_{\substack{z \leqq 2 n R_{\star} \\
z^{\prime} \geqq 2 n R_{\star}+t}} \frac{\rho(x, y, z) \rho\left(x^{\prime}, y^{\prime}, z^{\prime}\right)}{\left(\left(x-x^{\prime}\right)^{2}+\left(y-y^{\prime}\right)^{2}+\left(z-z^{\prime}-t\right)^{2}\right)^{1 / 2}} d \eta d \eta^{\prime} \\
& +\int_{\mathbb{R}^{3}} \rho_{t}^{1} B \rho_{0}^{2} .
\end{aligned}
$$

Using the fact that $\rho \equiv 0$ in $Z_{n}$ and the second integral above is $t$-independent we easily see,

$$
\left.\frac{d}{d t} J\left(\rho_{t}\right)\right|_{t=0}=\int_{\substack{z \leqq 2 n R_{\star} \\ z^{\prime} \geqq 2 n R_{\star}}} \frac{\rho(x, y, z) \rho^{\prime}\left(x^{\prime}, y^{\prime}, z^{\prime}\right)\left(z-z^{\prime}\right)}{\left(\left(x-x^{\prime}\right)^{2}+\left(y-y^{\prime}\right)^{2}+\left(z-z^{\prime}\right)^{2}\right)^{3 / 2}} d \eta d \eta^{\prime}
$$


Next we observe that the integrand above is non-positive in the region of integration. Further $\rho$ is a critical point of $J(\rho)$, thus the integral on the right vanishes. This forces $\rho \equiv 0$ for either $z \leqq 2 n R_{\star}$ or $z \geqq 2 n R_{\star}$. This proves claim (B) and hence Lemma (3.1) is fully established.

Combining the conclusions of Lemma (2.1) and Lemma (3.1) we easily have Theorem 1.

We now prove Theorem 2.

Proof of Theorem 2. As before set $u=\lambda+\frac{1}{2} \omega^{2} r^{2}+B \rho$. Then on the set where $\rho>0$ by $(0.6)$ we have $A^{\prime}(\rho)=u$. By Lemma (1.4) this gives us $\rho=\Phi(u)$. Let $\Gamma_{j}$ denote a connected component of the set where $\rho>0$. Differentiating the formula for $u$ we see that in each component $\Gamma_{j}$ we must necessarily have $\Delta u=3 \omega^{2}-4 \pi \rho$. Thus $u$ satisfies the boundary value problem,

$$
\begin{aligned}
\Delta u & =3 \omega^{2}-4 \pi \Phi(u), \quad \text { on } \Gamma_{j}, \\
\left.u\right|_{\partial \Gamma_{j}} & =0 .
\end{aligned}
$$

We now claim that inside $\Gamma_{j}$ we can find a ball of radius $A_{0}\left(\omega_{0}\right)$. To see this first note by Lemma (1.2) and (2.5), $\|\nabla B \rho\| \leqq C_{1}$. Thus in $\Gamma_{j}$ since $r \leqq d_{0}$ (by Lemma (2.1)) we also have $\|\nabla u\|_{\infty} \leqq C_{0}\left(\omega_{0}\right)$. Now suppose we can find a sequence of components of $\Gamma_{j}$ such that the diameters of the largest balls contained in $\Gamma_{j}$ tend to zero. Since $u$ vanishes on $\partial \Gamma_{j}$, and the gradient of $u$ is controlled, it means that given $\tau>0$ one will have $u<\tau$ as soon as $\sup _{x \in \Gamma_{,}} \operatorname{dist}\left(x, \partial \Gamma_{j}\right)<C_{0} \tau$. But this means that on such components, by Lemma (1.3) we have $\Phi(u)<C_{0} \tau^{1 /(\gamma-1)}$. But this means for $\omega \geqq \omega_{0}$, we have $3 \omega^{2}-4 \pi \Phi(u) \geqq 0$. Thus from (3.3) $u$ is subharmonic in $\Gamma_{j}$ and because $A^{\prime}(\rho)=u$ in $\Gamma_{j}$ we also have $u \geqq 0$ in $\Gamma_{j}$. Applying the maximum principle we conclude that $u \equiv 0$ in $\Gamma_{j}$. So our claim is proved. In particular we have

$$
\left|\Gamma_{j}\right| \geqq C_{1} A_{0}^{3} .
$$

But by Theorem 1 the support is contained in a ball of radius $\sigma$. Thus if $\Gamma_{1} \cdots \Gamma_{k}$ is an enumeration of the connected components of $\rho>0$ we see from (3.4) that we should have,

$$
k \leqq C_{0}\left(\frac{\sigma}{A_{0}}\right)^{3} .
$$

This establishes Theorem 2.

Acknnowledgements. Sagun Chanillo was supporterd by NSF grant DMS-9202051. YanYan Li was supported by a grant from the Alfred P. Sloan Foundation and by NSF grant DMS-9104293. S.C. is also indebted to Michael Kiessling for several comments on the physical viability of the rotating star model considered in this paper. Y.Y.L. would like to thank Professor D. Christodoulou for suggesting to him to study the compressible fluid model of uniformly rotating stars a few years ago and for many useful conversations since then. We also thank the referees for several comments which helped the presentation and simplification of the paper.

\section{References}

[A1] Auchmuty, J.F.G.: Existence of equilibrium figures. Arch. Rational Mech. Anal. 65, 249-261 (1977) 
[A2] Auchmuty, J.F.G.: The global branching of rotating stars. Arch. Rational Mech. Anal. 114, 179-194 (1991)

[AB1] Auchmuty, J.F.G., Beals, R.: Variational solutions of some non-linear free boundary problems. Arch. Rational Mech. Anal. 43, 255-271 (1971)

[AB2] Auchmuty, J.F.G., Beals, R.: Models of rotating stars. Astrophysical J., 165, 79-82 (1971)

[C1] Chandrasekhar, S.: An Introduction to the Study of Stellar Structure, New York: Dover Publications, 1992

[C2] Chandrasekhar, S.: Ellipsoidal Figures of Equilibrium, New York, Dover Publications, 1992

[C3] Chandrasekhar, S.: Ellipsoidal figures of equilibrium-an historical account. Commun. Pure Appl. Math. 20, 251-265 (1967)

[C4] Chandrasekhar, S.: The maximum mass of ideal white dwarfs. Astrophysical J. 74, 81 (1931)

[CF1] Caffarelli, L., Friedman, A.: The shape of axisymmetric rotating fluid. J. Func. Anal. 35, 100-142 (1980)

[CF2] Caffarelli, L., Friedman, A.: The free boundary in the Thomas-Fermi atomic model. J. Diff. Eqns. 32, 335-356 (1979)

[FT1] Friedman, A., Turkington, B.: Asymptotic estimates for an axisymmetric rotating fluid. J. Funct. Anal. 37, 136-163 (1980)

[FT2] Friedman, A., Turkington, B.: Existence and dimensions of a rotating white dwarf. J. Diff. Eqns. 42, 414-437 (1981)

[FT3] Friedman, A., Turkington, B.: The oblateness of an axisymmetric rotating fluid. Indiana Univ. Math. J. 29, 777-792 (1980)

[K] Kopal, Z.: Figures of Equilibrium of Celestial Bodies, Madison, WI: Univ. of Wisconsin Press, 1960

[L] Lamb, H.: Hydrodynamics. 6th. edn., Cambridge, Cambridge Univ. Press, 1932

[Li] Li, Y.Y.: On uniformly rotating stars. Arch. Rational Mech. Anal. 115, 367-393 (1991)

[LY] Lieb, E., Yau, H.T.: The Chandraskehar theory of stellar collapse as the limit of quantum mechanics. Commun. Math. Phys. 112, 147-174 (1987)

[Lio] P.L. Lions, minimization problems in $\mathrm{L}^{\prime}\left(\mathbb{R}^{3}\right)$ and applications to free boundary problems. Vol. II (Pavia, 1979), 385-399, Ist. Naz. Alta Mat. Francesco Severi, Rome, 1980

[P] Poincaré, H.: Figures d'Équilibre d'une Mass Fluide, Paris: Gauthier-Villars, 1901

[W] Wavre, R.: Figures Planétaires et Geodésie. Paris: Gauthier-Villars, 1932 